\title{
Teilnahmeanreiz mit Grenzen
}

\section{Gemessen an den Teilnehmerzahlen scheint die europäische Öko-Audit-Verord- nung (EMAS) kein Erfolg zu sein (1). Wie könnte eine Teilnahme für Unterneh- men attraktiver gestaltet werden? Ein möglicher Anreiz besteht in der Gewäh- rung von Vollzugserleichterungen für auditierte Unternehmen.}

D Von Frank Wätzold eutschland ist der einzige größere EUMitgliedsstaat, in dem sich eine signifikante Zahl von Unternehmen für die Beteiligung an EMAS entschieden hat und in dem (noch) mehr Unternehmen am Öko-Audit-System als an der internationalen UmweltmanagementsystemNorm ISO 14001 teilnehmen (vgl. Tab. 1). Vor diesem Hintergrund war es ein Ziel der Überarbeitung der Öko-Audit-Verordnung, die Teilnahme an EMAS für Unternehmen attraktiver zu gestalten. Als einen Anreiz fordert die neue Verordnung daher explizit in Artikel 10 die Mitgliedstaaten auf zu prüfen, ob der EMAS-Registrierung bei der Durchführung und Durchsetzung von Umweltvorschriften Rechnung getragen werden kann (2). Vollzugserleichterungen für Unternehmen mit einem standardisierten Umweltmanagementsystem lassen sich rechtfertigen, weil die Wahrscheinlichkeit, dass solche Unternehmen umweltgesetzliche Anforderungen erfüllen, unter anderem aufgrund eines besser entwickelten betrieblichen Umweltschutzes größer ist als bei anderen Unternehmen (3).

Im Folgenden wird untersucht, welchen Einfluss dieser Anreiz auf die Beteiligung an EMAS voraussichtlich haben wird. Zu diesem Zweck werden
Erfahrungen mit entsprechenden Maßnahmen in Deutschland, Frankreich, den Niederlanden und dem Vereinigten Königreich ausgewertet (4).

\section{Deutschland: Bayern vs. NRW}

In Deutschland wird über die Ausgestaltung des umweltgesetzlichen Vollzugs überwiegend auf Landesebene entschieden. Inzwischen haben alle Bundesländer Vollzugserleichterungen für ökoauditierte Unternehmen eingefïhrt. Diese Erleichterungen unterscheiden sich jedoch erheblich bezüglich ihres Umfangs sowie des institutionellen Rahmens, in den sie eingebettet sind. Exemplarisch seien die Bundesländer Bayern und Nordrhein-Westfalen (NRW) betrachtet.

In Bayern haben sich im Rahmen des am 23.0ktober 1995 verabschiedeten und inzwischen erneuerten Umweltpakts Bayern die Unternehmen freiwillig unter anderem dazu verpflichtet, dass sich mindestens 500 bayerische Unternehmen bis Oktober 2000 am Öko-Audit-System beteiligen. Im Gegenzug hat die Staatsregierung relativ umfangreiche Vollzugserleichterungen für öko-auditierte Betriebe zugesagt (5). Beide Seiten haben die hier genannten Zusagen erfüllt. Während die Staatsregierung in Zusammenarbeit mit dem bayerischen Verband der chemischen Industrie umfangreiche Deregulierungsmaßnahmen erarbeitet

Tabelle 1: Teilnehmerzahlen an EMAS und ISO 14001 (Januar 2001)

\begin{tabular}{|l|l|r|r|r|r|}
\hline & \multicolumn{2}{|c|}{ Tabelle 1: Teilnehmerzahlen an EMAS und ISO 14001 (Januar 2001) } \\
\hline & $\begin{array}{l}\text { Anzahl potenzieller Teilnehmer } \\
\text { (Unternehmen des verarbeiten- } \\
\text { den Sektors mit mehr als } \\
\text { 20 Mitarbeitern) }\end{array}$ & \multicolumn{3}{|c|}{ EMAS } & \multicolumn{2}{|c|}{ IS014001 } \\
\cline { 3 - 6 } & $\begin{array}{l}\text { Anzahl } \\
\text { registrierter } \\
\text { Teilnehmer }\end{array}$ & $\begin{array}{l}\text { In \% der } \\
\text { potenziellen } \\
\text { Teilnehmer }\end{array}$ & $\begin{array}{l}\text { Anzahl } \\
\text { zertifizierter } \\
\text { Teilnehmer }\end{array}$ & $\begin{array}{l}\text { In \% der } \\
\text { potenziellen } \\
\text { Teilnehmer }\end{array}$ \\
\hline $\begin{array}{l}\text { Vereinigtes } \\
\text { Königreich }\end{array}$ & 29.608 & 122 & 0,41 & 1.400 & 4,73 \\
\hline Deutschland & 37.413 & 2.632 & 7,03 & 2.400 & 6,41 \\
\hline Frankreich & 24.671 & 39 & 0,16 & 802 & 3,25 \\
\hline Niederlande & 6.404 & 26 & 0,41 & 800 & 12,50 \\
\hline $\begin{array}{l}\text { Alle EU-Mit- } \\
\text { gliedsstaaten }\end{array}$ & - & 3.800 & - & 9.812 & - \\
\hline
\end{tabular}

Quelle: eigene Berechnungen auf Basis von Eurostat - New Cronos Datenbank 12/98 und www.ecology.or.jp/isoworld/english/ana- und mit Hilfe von Verwaltungsvorschriften umgesetzt hat, waren bereits im Oktober 1999, ein Jahr früher als geplant, 500 Unternehmen nach EMAS zertifiziert.

In NRW wurden Vollzugserleichterungen für ökoauditierte Unternehmen in geringerem Umfang gewährt als in Bayern. Der im Mai 1998 verabschiedete Substitutionserlass beschäftigte sich ausschließlich mit Immissionsschutz und wies die Vollzugsbehörden an, ihren Entscheidungsspielraum bei der Überwachung so zu nutzen, dass nach EMAS zertifizierte Unternehmen weniger häufig kontrolliert werden und ihnen Entlastungen bei Dokumentations- und Informationspflichten gewährt werden.

Vergleicht man die tatsächlichen Teilnahmezahlen, so ergibt sich mit Stand März 2000 in Bayern eine Beteiligung von gut 8 Prozent der potenziellen Teilnehmer, während dieser Anteil in NRW nur 5 Prozent beträgt. Da die weiter gehenden Vollzugserleichterungen und die Einbindung des Öko-Audit-Systems in eine umfangreiche freiwillige Vereinbarung die einzigen signifikanten Unterschiede bei den Anreizen sind, die die Entscheidung von Unternehmen an einer EMAS-Beteiligung determinieren (6), lässt sich die Schlussfolgerung ziehen, dass diese beiden Faktoren für die höheren bayerischen Teilnehmerzahlen entscheidend sind.

Beide Bundesländer haben lange Zeit Vollzugserleichterungen ausschließlich EMAS-zertifizierten Unternehmen gewährt. Die Position der Länder in dieser Frage ist aber in einem Veränderungsprozess begriffen. Beispielsweise betrachtet in jüngster Zeit die bayerische Staatsregierung die Teilnahme an ISO 14001 und EMAS im Hinblick auf Vollzugserleichterungen als gleichwertig, wenn sich nach ISO 14001 zertifizierte Unternehmen verpflichten, den Umweltschutz kontinuierlich zu verbessern, alle umweltgesetzlichen Vorschriften einzuhalten sowie die Öffentlichkeit regelmäßig über ihre Umweltaktivitäten zu informieren.

\section{Frankreich: Marketing?}

Französische Unternehmen haben seit der Einführung der Öko-Audit-Verordnung gefordert, dass die freiwillige Teilnahme an EMAS durch Vollzugserleichterungen belohnt werden sollte. Das französische Umweltministerium, das den Vollzugsbehörden vorsteht, hat bisher jedoch keine formalen Vollzugserleichterungen gewährt. Das Ministerium begründet seine Entscheidung damit, dass alle Firmen vor dem Gesetz gleichberechtigt behandelt werden sollen, 
dass nicht alle registrierten Unternehmen ein vergleichbar hohes Umweltschutzniveau erreichen und dass in der Öko-Audit-Verordnung nicht alle Bereiche des betrieblichen Umweltschutzes berücksichtigt werden, zum Beispiel ist das Risikomanagement ausgeklammert. Das französische Umweltministerium hat auch EMAS nie als ein Element der staatlichen Umweltpolitik angesehen, sondern lediglich als Instrument, das den Firmen erlaubt, mit ihren Umweltleistungen zu werben. Informell werden in Frankreich in verschiedenen Verwaltungsbezirken jedoch Unternehmen mit einem standardisierten Umweltmanagementsystem (unabhängig davon ob nach EMAS oder ISO 14001) Vollzugserleichterungen gewährt.

\section{- Niederlande: Eigenverantwortung}

Schon der niederländische Umweltpolitikplan von 1989 sah unter anderem die Förderung des Einsatzes von Umweltmanagementsystemen in Unternehmen sowohl direkt, beispielsweise durch Informationsmaßnahmen, als auch indirekt über Abkommen zwischen der Regierung und einzelnen Branchen vor. In diesen Abkommen verpflichteten sich die Branchen, bestimmte Umweltziele zu erreichen. Umweltmanagementsysteme wurden hierbei als Mittel zur Erreichung dieser Ziele angesehen aber auch als Mittel um den notwendigen unternehmensinternen Wandel zu überwachen.

Grundsätzlich unterscheiden die niederländischen Vollzugsbehörden zwischen pro-aktiven Unternehmen und „Nachzüglern“. Als pro-aktiv gelten Unternehmen, die überdurchschnittliche Umweltschutzbemühungen zeigen, und von denen erwartet werden kann, dass sie aus eigener Verantwortung heraus Umweltschutz betreiben. Solchen Unternehmen ist es gestattet, Überwachungsmaßnahmen weitgehend eigenverantwortlich durchzuführen. Weiterhin werden bei Genehmigungsverfahren für pro-aktive Unternehmen eher Ziele als detaillierte Regelungen festgelegt, um den Unternehmen einen möglichst weit gehenden Handlungsspielraum zu lassen. Ein zertifiziertes Umweltmanagementsystem führt nicht automatisch dazu, dass die Behörden ein Unternehmen als pro-aktiv einschätzen. Es ist dafür aber ein wichtiges Hilfsmittel. Hierbei macht es aus Sicht der Behörden keinen Unterschied, ob ein Unternehmen nach EMAS oder ISO 14001 zertifiziert ist.

\section{- Vereinigtes Königreich: So what?}

Das britische Umweltministerium sowie die oberste Vollzugsbehörde, seit 1997 die Environmental Agency, haben weit gehende Vollzugserleichterungen für Unternehmen mit einem zertifizierten Umweltmanagementsystem bis vor kurzem abgelehnt. Bisher ist die Teilnahme eines Unternehmens an EMAS oder ISO 14001 nur einer von vierzehn Faktoren, die unter dem seit 1997 bestehenden ,Operator and Risk Appraisal System“ helfen sollen, potenzielle Umweltgefährdungen durch Unternehmen und damit die notwendige Überwachungsintensität zu bestimmen. Seit Oktober 1999 gibt es jedoch innerhalb der Umweltbehörde eine Diskussion darüber, ob bei der Überwachung verstärkt der unternehmensinternen Eigenkontrolle, zum Beispiel durch Umweltmanagementsysteme, vertraut werden soll. Welche Folgen sich aus dieser Diskussion ergeben, ist zur Zeit noch nicht absehbar. Hervorzuheben ist jedoch, dass es keine Anzeichen gibt, die bisherige Politik, die Teilnahme an EMAS und ISO 14001 als gleichwertig in Bezug auf Vollzugserleichterungen zu behandeln, aufzugeben.

\section{- Schlussfolgerungen}

Die Aufforderung in der revidierten Öko-AuditVerordnung, Vollzugserleichterungen für ökoauditierte Unternehmen zu prüfen, ist nichts Neues, sondern etwas, das bereits diskutiert und auch in unterschiedlichem Umfang verwirklicht worden ist. Selbst wenn es zu einer Ausweitung von Vollzugserleichterungen kommen sollte, dürfte vor dem Hintergrund, dass in England, Frankreich sowie den Niederlanden die Teilnahme an EMAS und ISO 14001 in dieser Hinsicht als gleichwertig angesehen wird, sich in Deutschland ein ähnlicher Trend abzeichnet und auch dort inzwischen viele Unternehmen ISO 14001 bevorzugen, die Folge eher eine erhöhte Teilnahme an ISO 14001 als an EMAS sein.

Anhand des Vergleichs zwischen Bayern und NRW lässt sich ableiten, dass signifikante Vollzugserleichterungen und die Einbettung des Öko-AuditSystems in eine umfassende freiwillige Vereinbarung die Teilnahmeentscheidungen der Unternehmen für ein standardisiertes Umweltmanagementsystem positiv beeinflussen können. Diese Schlussfolgerung wird durch die Analyse der anderen Länder unterstützt. Sowohl in Frankreich als auch in England, wo die Teilnehmerzahlen nicht nur an EMAS sondern auch an ISO 14001 vergleichsweise niedrig sind, werden relativ geringe Vollzugserleichterungen gewährt. Im Gegensatz hierzu sind in den Niederlanden, wo - ähnlich wie in Bayern - substanzielle Vollzugserleichterungen gewährt werden, die in einen umfassenden Umweltpakt eingebunden sind, die Teilnehmerzahlen an ISO 14001 relativ hoch (vgl. Tab. 1). Zu beachten ist jedoch, dass erst eine umfassendere Analyse zu sagen erlaubt, welchen Einfluss Vollzugserleichterungen und welchen Einfluss andere Faktoren auf die Teilnahmeentscheidung der Unternehmen in den betrachteten Ländern haben.

\section{Anmerkungen}

(1) Eine solche Sichtweise reflektiert den Erfolg der Öko-Audit-Verordnung aber nur unzureichend, vgl. dazu ausführlich Wätzold, F./ Bültmann, A.: The implementation of EMAS in Europe: A case of competition between standards for environmental management systems. In: Glachant, M. (Ed.): Implementing European Environmental Policy: the Impacts of Directives in the Member States, Cheltenham (im Druck). (2) Vgl. zur Novellierung der Verordnung auch den Beitrag von Jens Pape in Ökologisches Wirtschaften 1/01, S. 6-7. Ob aus normativer Sicht die Teilnahme eines Unternehmens an EMAS oder ISO 14001 zu präferieren ist, hängt von den jeweiligen gesellschaftlichen Kosten und Nutzen der Teilnahmeentscheidung ab. Vgl. Wätzold, F./ Bültmann, A.: Wie viele Unternehmen sollen an einem Umweltmanagementstandart teilnehmen? UFZ-Diskussionspapier 7/00, Leipzig. (3) Vgl. für eine ausführliche Begründung Wätzold, F./ Bültmann, A./ Eames, M. / Lulofs, K./ Schucht, S.: EMAS and Regulatory Relief: Lessons from National Experiences, in: European Environment, Vol. 11/1 (2001), S. 37-48 sowie die dort angegebene (auch kontroverse) Literatur. (4) Die Informationen für die Auswertung entstammen Bültmann, A./ Wätzold, F.: The implementation of national and European environmental legislation in Germany. Three case studies. UFZ research report 20/2000. Leipzig 2000; Eames, M.: Implementation of the EMAS Regulation in the United Kingdom. SPRU research report, Brighton 2000; Lulofs, K.: Implementation of EMAS in the Netherlands, Research Paper 2000-B-5, Cerna, Paris 2000 und Schucht, S.: The implementation of the EMAS Regulation in France. Research Paper 2000-B-2, Cerna, Paris 2000.

(5) Vgl. dazu www.umweltministerium.bayern.de/agenda/ wirtsch/wirtsch.htm. und Petschow, Ulirch: Der Umweltpakt Bayern, in: Ökologisches Wirtschaften 1/1996, S. 4. (6) Vgl. dazu Wätzold et al. a.a.0. (Anm. 3).

\section{Der Autor}

Dr. Frank Wätzold ist Wissenschaftler am UFZ-Umwelfforschungszentrum Leipzig Halle, Sektion Ökonomie, Soziologie und Recht.

Kontakt: UFZ, Permoserstr. 15, 04318 Leipzig,

Tel. 0341/ 235-2670, Fax -2825,

E-mail: waetzold@ulok.ufz.de 
(c) 20I0 Authors; licensee IÖW and oekom verlag. This is an article distributed under the terms of the Creative Commons Attribution Non-Commercial No Derivates License (http://creativecommons.org/licenses/by-nc-nd/3.o/), which permits unrestricted use, distribution, and reproduction in any medium, provided the original work is properly cited. 